\title{
Being red, blue and green: the genetic basis of coloration differences in the strawberry poison frog (Oophaga pumilio)
}

\author{
Ariel Rodríguez ${ }^{1 *}$ (D) Nicholas I. Mundy², Roberto Ibáñez ${ }^{3,4}$ and Heike Pröhl ${ }^{1}$
}

\begin{abstract}
Background: Animal coloration is usually an adaptive attribute, under strong local selection pressures and often diversified among species or populations. The strawberry poison frog (Oophaga pumilio) shows an impressive array of color morphs across its distribution in Central America. Here we quantify gene expression and genetic variation to identify candidate genes involved in generating divergence in coloration between populations of red, green and blue O. pumilio from the Bocas del Toro archipelago in Panama.
\end{abstract}

Results: We generated a high quality non-redundant reference transcriptome by mapping the products of genome-guided and de novo transcriptome assemblies onto a re-scaffolded draft genome of O. pumilio. We then measured gene expression in individuals of the three color phenotypes and identified color-associated candidate genes by comparing differential expression results against a list of a priori gene sets for five different functional categories of coloration - pteridine synthesis, carotenoid synthesis, melanin synthesis, iridophore pathways (structural coloration), and chromatophore development. We found 68 candidate coloration loci with significant expression differences among the color phenotypes. Notable upregulated examples include pteridine synthesis genes spr, $x d h$ and pts (in red and green frogs); carotenoid metabolism genes bco2 (in blue frogs), scarb1 (in red frogs), and guanine metabolism gene psat1 (in blue frogs). We detected significantly higher expression of the pteridine synthesis gene set in red and green frogs versus blue frogs. In addition to gene expression differences, we identified 370 outlier SNPs on 162 annotated genes showing signatures of diversifying selection, including eight pigmentation-associated genes.

Conclusions: Gene expression in the skin of the three populations of frogs with differing coloration is highly divergent. The strong signal of differential expression in pteridine genes is consistent with a major role of these genes in generating the coloration differences among the three morphs. However, the finding of differentially expressed genes across pathways and functional categories suggests that multiple mechanisms are responsible for the coloration differences, likely involving both pigmentary and structural coloration. In addition to regulatory differences, we found potential evidence of differential selection acting at the protein sequence level in several color-associated loci, which could contribute to the color polymorphism.

Keywords: Coloration genetics, Pigments, Gene expression, SNPs, Poison frog

\footnotetext{
* Correspondence: ariel.rodriguez@tiho-hannover.de

${ }^{1}$ Institute of Zoology, University of Veterinary Medicine of Hannover,

Bünteweg 17, 30559 Hannover, Germany

Full list of author information is available at the end of the article
}

\section{$\triangle B M C$}

(c) The Author(s). 2020 Open Access This article is licensed under a Creative Commons Attribution 4.0 International License, which permits use, sharing, adaptation, distribution and reproduction in any medium or format, as long as you give appropriate credit to the original author(s) and the source, provide a link to the Creative Commons licence, and indicate if changes were made. The images or other third party material in this article are included in the article's Creative Commons licence, unless indicated otherwise in a credit line to the material. If material is not included in the article's Creative Commons licence and your intended use is not permitted by statutory regulation or exceeds the permitted use, you will need to obtain permission directly from the copyright holder. To view a copy of this licence, visit http://creativecommons.org/licenses/by/4.0/. The Creative Commons Public Domain Dedication waiver (http://creativecommons.org/publicdomain/zero/1.0/) applies to the data made available in this article, unless otherwise stated in a credit line to the data. 


\section{Background}

Animal coloration plays important roles in intra- and interspecific communication, thermoregulation, predator avoidance and other ecological interactions with direct impact on individual fitness. Color phenotypes are often under strong local selection pressures and can be strikingly different among related species or populations [1$3]$. For its functional significance, diversity, and the relative ease of obtaining comparable measurements, coloration is one of the most tractable traits in evolutionary research $[4,5]$.

The extended color palette exhibited by animals is produced by a combination of the selective absorption of light by different types of pigments and light scattering on reflective structures such as purine crystals or keratin [6-8]. Until very recently, knowledge of the genetic basis of vertebrate coloration has focused on a few species of mammals, birds and fish and strongly biased towards melanin-based coloration [9]. This situation is rapidly changing with the increase in power and affordability of genomic sequencing technologies.

In amphibians, reptiles and fish, integumentary coloration is produced by three main types of chromatophore cell: melanophores, xanthophores and iridophores [10]. Melanophores synthesize brown/black melanin pigment, xanthophores express yellow to red pteridine and/or carotenoid pigments, and iridophores produce reflective guanine crystals contributing to structural coloration. The typical arrangement of these cells is in a threelayered sandwich, with xanthophores overlying iridophores, and melanophores in the basal position, forming a "dermal chromatophore unit" [11].

Across vertebrates, the genetics of melanin-based coloration is relatively well studied and multiple genes involved in natural variation in melanin coloration have been identified in case studies on mammals, reptiles, birds and fishes [5]. In contrast, comparatively, little is known about the genetic basis of carotenoid and pteridine based pigmentation. The biosynthetic pathway for pteridine synthesis, based on guanosine triphosphate (GTP), was elucidated in zebrafish [12], and later generalized for vertebrates [13]. Unlike melanin and pteridines, carotenoids cannot be produced de novo by vertebrates but have to be obtained by ingestion, and hence their availability is environmentally-dependent [14]. Assimilation, modification and accumulation of carotenoids in their target tissues involve numerous steps and results in a large number of molecular interactions impacting many aspects of vertebrate physiology [15, 16]. Variation in guanine-based structural coloration produced by iridophores is poorly studied, with most work being performed in zebrafish [14].

In contrast to fish, birds and mammals little is known about the molecular and genetic basis of coloration in amphibians. One of the most remarkable examples of natural intra-specific polymorphism in amphibians is the small and visually conspicuous strawberry poison frog Oophaga pumilio (Schmidt, 1857), a member of Dendrobatidae that inhabits tropical rain forests in Central America. While the ancestral and most frequent color phenotype is bright red $[17,18]$, a broad array of color morphs have evolved on the mainland and especially the islands of the Bocas del Toro archipelago in Panama [19]. The dorsal coloration of these frogs is considered aposematic since alkaloids from their insect prey are sequestered in skin glands as chemical protection to discourage predators such as birds [20-22]. Multiple scenarios have been proposed to explain the staggering diversity of color phenotypes in this frog. Summers et al. [23] compared the phylogeography of the color polymorphic O. pumilio with two sympatric, color monomorphic species and inferred that sexual selection was involved in driving the rapid divergence in color and pattern between populations of $O$. pumilio. In this species, females make an important parental care investment and are extremely choosy with their mates displaying a significant preference for brightly colored males of their own color morph [24-28]. Maan and Cummings [29] demonstrated that color diversity in $O$. pumilio is also tightly linked to variation in toxicity and proposed that the polymorphism observed in Bocas del Toro might derive from an interaction between environmental heterogeneity of alkaloid availability, varying predation pressure and sexual selection by females. On the other hand, coalescent simulations suggest that, due to recent population expansions and the small island population sizes, genetic drift might have played a major role in the diversification of color across populations [30]. More recently, Yang et al. [31] analyzed female attraction and male aggression experiments in a crossfostering study and found a combination of rival and sexual imprinting in these frogs, which could reduce gene flow between individuals that bear divergent mating traits and set the stage for speciation by sexual selection.

While the ecological and evolutionary factors contributing to the fascinating color divergence in Oophaga pumilio populations have been investigated from different angles, the contribution of molecular processes has been neglected so far. Breeding experiments show that offspring of crosses between color phenotypes typically display a mixture of parental coloration but with color pattern if one parent showed color pattern, which is suggestive of a single locus control of color pattern and a polygenic control of coloration [32]. A recent study on Dendrobates auratus, another species of Dendrobatidae, identified a large number of differentially expressed genes likely responsible for coloration differences, some 
of which showed single-nucleotide polymorphism (SNP) variation between color morphs [33], lending support to the polygenic control hypothesis.

In order to obtain a molecular perspective on the genomic basis of color polymorphism in amphibians, we herein studied three plain colored and strikingly divergent morphs of $O$. pumilio showing blue, red and green dorsal skin color. We used a combination of methods for gene expression quantification and single-nucleotide polymorphism (SNP) detection using RNA-seq data obtained from dorsal skin of wild-captured animals to identify candidate genes related to color variation. We hypothesized that red and green frogs would have upregulated pteridine and/or carotenoid pathways, whereas blue frogs would have upregulated iridophore and/or melanogenic pathways involved in structural coloration.

\section{Results}

\section{Draft genome re-scaffolding}

The published Oophaga pumilio reference genome is a heavily fragmented draft, containing 7,182,834 scaffolds with $\mathrm{N} 50=79,909$ and largest contig $(\mathrm{LC})=0.9 \mathrm{Mb}$. Rescaffolding of this draft with paired RNA-Seq reads in P_RNA software resulted in substantial improvements in contiguity $(631,034$ scaffolds, N50 $=116,040, \mathrm{LC}=1.7$ $\mathrm{Mb})$. This re-scaffolded genome had an improved BUSCO score $(85.5 \%$ of completeness vs the original $76.6 \%$ ) and was therefore used for subsequent analyses. Statistics of the two assemblies are provided in SMTable 1.

\section{Reference transcriptome assembly and annotation}

The genome-guided and de novo assemblies resulted in $1,080,547(\mathrm{~N} 50=876)$ and 980,876 (N50=1024) transcripts respectively. A large fraction of these transcripts $(2,031,063 ; 98.5 \%)$ aligned to the re-scaffolded draft genome and were combined in a non-redundant and comprehensive PASA transcriptome including 903,736 transcript sequences derived from 617,432 PASA clusters in the genome (representing gene structures from transcriptionally active regions which we tentatively assume as genes). The BUSCO scoring of this reference transcriptome indicated $94.5 \%$ completeness, $3.0 \%$ fragmentation, and $2.5 \%$ missing genes of the 2586 vertebrate bench-marking genes. This reference transcriptome includes 274,940 ORF-containing transcripts, 161, 968 of which had positive blast hits against the UniProt database. Of these, 92,442 transcripts were identified as UniProt orthologs and 69,526 as paralogs. The coding transcripts originate from 35,953 distinct PASA clusters (genes) in the reference genome including 12,821 orthologs to sequences in UniProt database and 23,132 paralogs. Subsequent functional interpretations of results were restricted to the subset of orthologous coding genes.

\section{Differential expression analyses}

A total of 24,390 coding genes were expressed in the $O$. pumilio skin samples. A PCA analysis on expression levels in the fifteen samples showed strong clustering within populations and divergence between populations (Fig. 1). Sleuth analyses identified 2639 differentially expressed (DE) genes between the three color morphs (SMTable 2). Of these, 1445 were orthologs to Uniprot genes and the inspection of the expression profiles identified six DE gene clusters with functions related to angiogenesis, the gonadotropin-releasing hormone receptor, and multiple signaling pathways (SMFigure 1, SMTable 3). We identified $68 \mathrm{DE}$ genes linked to pigment production, structural coloration in iridophores, and pigment-cell differentiation in previous studies (Table 1.). Seven genes were DE in the carotenoid metabolism pathway; $r d h 10$ and $b c o 2$ were up-regulated in blue frogs, while dgat 2 and $d h r s 3$ were up-regulated in green frogs. In red frogs the aldh1a1 enzyme was downregulated while scarb1 was up-regulated in comparison to blue and green frogs (Table 1, Fig. 2). Three DE genes were found in the pteridine synthesis pathway: spr was upregulated in red frogs, pts was up-regulated in green frogs, $x d h$ was up-regulated in red and green frogs, and no gene was up-regulated in blue frogs (Table 1, Fig. 2). Fourteen DE genes were found in the melanin synthesis pathway: oca 2 and plcb4 were up-regulated in blue frogs; the ctnnb1 gene was up-regulated in green frogs while wnt10b, gnai1, wnt9a, ep300, adcy6, raf1 and camk $2 g$ were up-regulated in green and red frogs (Table 1, Fig. 2). Four DE genes were found in the iridophore guanine synthesis pathway: psat1 was upregulated in blue frogs, $a d s l$ was up-regulated in green frogs, and $f h$ was upregulated in red frogs. Additionally, we found $40 \mathrm{DE}$ genes previously linked to chromatophore development and differentiation (Table 1, Fig. 2). Interesting candidates were $\operatorname{pax} 7$, and sox 9 , which were upregulated in green frogs; med1, med12, and myo5a, which were upregulated in red frogs; and dock7, hps1, hps3, hps4 and $s f 3 b 1$ which were up-regulated in blue frogs.

\section{Gene set enrichment and over-representation analyses}

Significant enrichment was detected in the genes of the pteridine synthesis pathway in red vs blue and green vs blue comparisons. The remaining pigment-synthesis gene sets showed no significant enrichment in any of the pair-wise comparisons (Table 2). Results of the overrepresentation analysis of the entire set of $1445 \mathrm{DE}$ genes indicated a total of 312 enriched gene ontology (GO) terms of different categories (biological process: 183, cellular component: 71 , and molecular function: 58 ; 

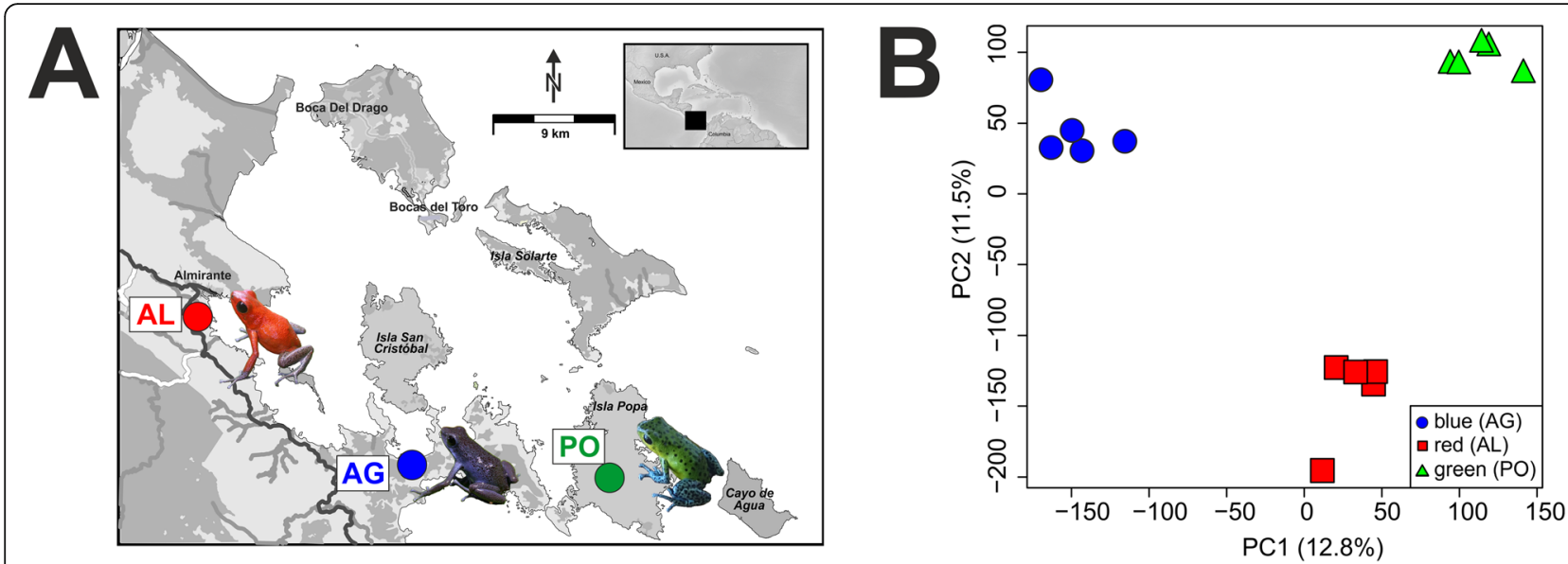

Fig. 1 Sampling scheme and general gene expression patterns. A) Geographic location of localities in the Bocas del Toro archipelago where Oophaga pumilio samples were obtained (AL, Almirante; AG, Aguacate; PO, Popa) and their associated color phenotypes (inset photos). B) Plot of the principal component analysis summarizing the expression pattern across samples of the three color phenotypes. The background map in A (๔ OpenStreetMap contributors) was created with open data cartography licensed under a Creative Commons Attribution-ShareAlike 2.0 license (CC BY-SA, https://www.openstreetmap.org/copyright)

SMTable 4). These include categories related to biological processes including: protein folding response, endoplasmic reticulum stress response, establishment or maintenance of cell polarity and amino acid and organonitrogen compound metabolic process. The gene ontology categories are localized in multiple cell compartments, from the cytoplasm to pigment granules, vesicles and the endoplasmic reticulum. Over-represented molecular functions included: RNA binding, oxidoreductase activity, protein binding, organic cyclic compound binding, small molecule binding, and chaperone binding. A graphical overview of the over-represented categories, as obtained from REVIGO, is presented in SMFigure 2.

\section{SNPs and signatures of selection}

A total of 1,917,067 SNPs were identified with the GATK pipeline in the assembled superTranscriptome of O. pumilio. Of these, 398,910 bi-allelic SNPs passed the sample and genotype coverage thresholds and were subjected to BayeScan analyses. Results identified a total of 370 outlier SNPs showing signature of directional selection with $F_{\text {st }}$ among the three populations ranging between 0.44 and 0.57 with an upper trace in the $F_{\text {st }}$ vs $q$-value plot representing the SNPs with maximal differentiation between color morphs - fixed ( 0 or 1$)$ allelic frequencies (Fig. 3a). The outlier SNPs were located in 762 transcripts, with twice as many outlier SNPs in $3^{\prime}$ untranslated regions ( $3^{\prime}$-UTR, 562, 39\%) compared to $5^{\prime}$ untranslated regions (5'UTR, 252, 17\%). Among outlier SNPs in the coding sequence (CDS, 637, 44\%) there were four times as many non-synonymous (512) compared to synonymous (127) variants (Fig. 3b). Outlier SNPs occurred on 162 annotated genes and 39\% of these
(65 genes) also showed significant expression differences which could represent functional SNPs under selection that affect gene expression (SMTable 5). Twelve of the outlier SNPs occurred on eight genes associated with pigmentation (Table 3) and, of these, kit showed the strongest signal, with four linked outlier SNPs, spanning $\sim 630 \mathrm{bp}$, fixed in blue frogs (Fig. 3c). One of these SNPs represented a non-synonymous substitution while the other three lay in the 3'UTR region (SMFigure 3). It is worth noting that no significant differences in expression were detected for this gene in Sleuth tests (SMFigure 4).

\section{Discussion}

Our results support a key role of regulatory control but also a potential role of single-nucleotide polymorphisms in shaping the observed differences in skin coloration in O. pumilio frogs of the Bocas del Toro archipelago. The multidimensional nature of the molecular basis for color diversity discovered in this study is not surprising, considering that skins of amphibians are multilayered, three-dimensional structures of several cell types that often contain multiple pigment types and structural features [34]. It is the interplay among the reflection and absorbance of light of different wavelengths with the presence and absence of certain pigments, aggregation or dispersion of pigment containing organelles (e.g. melanosomes) which determines the color phenotype of the animal $[34,35]$.

\section{Gene expression differences}

A great diversity of pteridines can be found in amphibian skin, including Oophaga, and these contribute to 
Table 1 Differentially expressed genes between blue, green and red color phenotypes of Oophaga pumilio previously linked to coloration

\begin{tabular}{|c|c|c|c|c|c|}
\hline rank & gene & transcripts & q-val & expression-pattern & pigmentation role \\
\hline 88 & dgat2 & 4 & 0.000 & GREEN > RED $=$ BLUE & carotenoid metabolism \\
\hline 102 & rdh16 & 4 & 0.000 & $\mathrm{BLUE}=\mathrm{GREEN}>\mathrm{RED}$ & carotenoid metabolism \\
\hline 145 & scarb1 & 1 & 0.000 & RED $>$ BLUE $>$ GREEN & carotenoid metabolism \\
\hline 371 & rdh10 & 2 & 0.000 & BLUE $>$ RED $=$ GREEN & carotenoid metabolism \\
\hline 691 & bco2 & 6 & 0.002 & BLUE $>$ RED $=$ GREEN & carotenoid metabolism \\
\hline 969 & dhrs3 & 1 & 0.005 & GREEN $>$ RED $=$ BLUE & carotenoid metabolism \\
\hline 1124 & aldhiar & 7 & 0.008 & $\mathrm{BLUE}=\mathrm{GREEN}>\mathrm{RED}$ & carotenoid metabolism \\
\hline 240 & adsl & 8 & 0.000 & GREEN $>$ RED $=$ BLUE & guanine synthesis in iridophores \\
\hline 525 & psat1 & 4 & 0.001 & BLUE $>$ RED $=$ GREEN & guanine synthesis in iridophores \\
\hline 1807 & gmps & 7 & 0.024 & RED $=$ GREEN $>$ BLUE & guanine synthesis in iridophores \\
\hline 1871 & fh & 5 & 0.025 & RED $>$ GREEN $>$ BLUE & guanine synthesis in iridophores \\
\hline 12 & camk2g & 6 & 0.000 & RED $=$ GREEN $>$ BLUE & melanin synthesis \\
\hline 91 & gnail & 7 & 0.000 & RED $>$ GREEN $>$ BLUE & melanin synthesis \\
\hline 98 & ctnnbl & 13 & 0.000 & GREEN $>$ RED $=$ BLUE & melanin synthesis \\
\hline 197 & raf1 & 8 & 0.000 & RED $=$ GREEN $>$ BLUE & melanin synthesis \\
\hline 481 & wnt9a & 3 & 0.001 & RED $>$ GREEN $>$ BLUE & melanin synthesis \\
\hline 521 & adcy6 & 4 & 0.001 & RED $>$ GREEN $>$ BLUE & melanin synthesis \\
\hline 771 & wnt11 & 3 & 0.003 & BLUE $>$ GREEN $>$ RED & melanin synthesis \\
\hline 797 & nras & 3 & 0.003 & $\mathrm{BLUE}=\mathrm{GREEN}>\mathrm{RED}$ & melanin synthesis \\
\hline 1718 & camk2d & 4 & 0.022 & $\mathrm{BLUE}=\mathrm{GREEN}>\mathrm{RED}$ & melanin synthesis \\
\hline 1939 & ep300 & 4 & 0.027 & RED $>$ GREEN $>$ BLUE & melanin synthesis \\
\hline 2036 & plcb4 & 9 & 0.031 & BLUE $>$ RED $=$ GREEN & melanin synthesis \\
\hline 2100 & wnt10b & 6 & 0.032 & RED $>$ GREEN $>$ BLUE & melanin synthesis \\
\hline 2571 & oca2 & 3 & 0.048 & BLUE $>$ RED $=$ GREEN & melanin synthesis \\
\hline 2620 & adcy3 & 2 & 0.050 & BLUE $>$ GREEN $>$ RED & melanin synthesis \\
\hline 119 & $x d h$ & 9 & 0.000 & RED $=$ GREEN $>$ BLUE & pteridine synthesis \\
\hline 853 & spr & 2 & 0.004 & RED $>$ GREEN $>$ BLUE & pteridine synthesis \\
\hline 975 & pts & 2 & 0.005 & GREEN $>$ RED $=$ BLUE & pteridine synthesis \\
\hline 1282 & sox9 & 4 & 0.010 & GREEN $>$ RED $=$ BLUE & chromatophore differentiation \\
\hline 167 & $\operatorname{atp} 12 a$ & 10 & 0.000 & BLUE $>$ RED $=$ GREEN & chromatophore differentiation \\
\hline 280 & hps3 & 14 & 0.000 & BLUE $>$ GREEN $>$ RED & chromatophore differentiation \\
\hline 1509 & hps4 & 5 & 0.016 & BLUE $>$ RED $=$ GREEN & chromatophore differentiation \\
\hline 2260 & hps1 & 8 & 0.037 & BLUE $>$ GREEN $>$ RED & chromatophore differentiation \\
\hline 2176 & myo5a & 2 & 0.034 & RED $>$ GREEN $>$ BLUE & chromatophore differentiation \\
\hline 1180 & slc7a11 & 2 & 0.009 & GREEN $>$ RED $=$ BLUE & chromatophore differentiation \\
\hline 316 & med1 & 7 & 0.000 & RED $>$ GREEN $>$ BLUE & chromatophore differentiation \\
\hline 32 & egfr & 7 & 0.000 & $\mathrm{BLUE}=\mathrm{GREEN}>\mathrm{RED}$ & chromatophore differentiation \\
\hline 55 & sult2b1 & 5 & 0.000 & BLUE $>$ RED $=$ GREEN & chromatophore differentiation \\
\hline 128 & $n f 1$ & 19 & 0.000 & RED $=$ GREEN $>$ BLUE & chromatophore differentiation \\
\hline 327 & gpr161 & 5 & 0.000 & RED $=$ GREEN > BLUE & chromatophore differentiation \\
\hline 337 & fos & 4 & 0.000 & RED $=$ GREEN > BLUE & chromatophore differentiation \\
\hline 453 & gnpat & 10 & 0.001 & BLUE $>$ RED $=$ GREEN & chromatophore differentiation \\
\hline 457 & $\mathrm{mpz} / 3$ & 1 & 0.001 & RED $>$ BLUE $>$ GREEN & chromatophore differentiation \\
\hline
\end{tabular}


Table 1 Differentially expressed genes between blue, green and red color phenotypes of Oophaga pumilio previously linked to coloration (Continued)

\begin{tabular}{|c|c|c|c|c|c|}
\hline rank & gene & transcripts & q-val & expression-pattern & pigmentation role \\
\hline 487 & srm & 5 & 0.001 & RED $>$ BLUE $>$ GREEN & chromatophore differentiation \\
\hline 491 & atp6r1h & 3 & 0.001 & RED $>$ BLUE > GREEN & chromatophore differentiation \\
\hline 572 & mfsd 12 & 5 & 0.001 & BLUE $>$ RED $=$ GREEN & chromatophore differentiation \\
\hline 611 & $\operatorname{cog} 4$ & 14 & 0.001 & BLUE $>$ RED $=$ GREEN & chromatophore differentiation \\
\hline 641 & $d s t$ & 11 & 0.002 & BLUE $>$ GREEN > RED & chromatophore differentiation \\
\hline 666 & oat & 4 & 0.002 & BLUE $>$ RED $=$ GREEN & chromatophore differentiation \\
\hline 722 & $s / c 24 a 4$ & 5 & 0.002 & $\mathrm{BLUE}=\mathrm{GREEN}>$ RED & chromatophore differentiation \\
\hline 813 & mbtps1 & 6 & 0.003 & BLUE $>$ GREEN > RED & chromatophore differentiation \\
\hline 1022 & mlana & 5 & 0.006 & BLUE $>$ RED $=$ GREEN & chromatophore differentiation \\
\hline 1053 & herc2 & 13 & 0.006 & RED $>$ GREEN $>$ BLUE & chromatophore differentiation \\
\hline 1130 & adrb1 & 4 & 0.008 & RED $>$ GREEN $>$ BLUE & chromatophore differentiation \\
\hline 1270 & casp3 & 3 & 0.010 & $\mathrm{BLUE}=\mathrm{RED}>\mathrm{GREEN}$ & chromatophore differentiation \\
\hline 1271 & dock7 & 2 & 0.010 & BLUE > GREEN > RED & chromatophore differentiation \\
\hline 1388 & atrn & 4 & 0.013 & RED $>$ BLUE $>$ GREEN & chromatophore differentiation \\
\hline 1457 & ecel & 6 & 0.015 & BLUE $>$ RED $=$ GREEN & chromatophore differentiation \\
\hline 2086 & mgrn1 & 3 & 0.032 & GREEN $>$ RED $=$ BLUE & chromatophore differentiation \\
\hline 2118 & med12 & 6 & 0.033 & RED $>$ GREEN $>$ BLUE & chromatophore differentiation \\
\hline 2141 & atp6riel & 3 & 0.033 & RED $>$ BLUE $>$ GREEN & chromatophore differentiation \\
\hline 2438 & edn3 & 5 & 0.043 & GREEN $>$ RED $=$ BLUE & chromatophore differentiation \\
\hline 2439 & gfpt1 & 4 & 0.043 & RED $=$ GREEN $>$ BLUE & chromatophore differentiation \\
\hline 2468 & pnp & 3 & 0.044 & $\mathrm{BLUE}=\mathrm{RED}>\mathrm{GREEN}$ & chromatophore differentiation \\
\hline 2500 & $s f 3 b 1$ & 6 & 0.045 & BLUE $>$ RED $=$ GREEN & chromatophore differentiation \\
\hline 2592 & $r t f 1$ & 1 & 0.049 & $\mathrm{BLUE}=\mathrm{RED}>\mathrm{GREEN}$ & chromatophore differentiation \\
\hline 2134 & impdh2 & 5 & 0.033 & BLUE $>$ RED $=$ GREEN & chromatophore differentiation \\
\hline 800 & pax7 & 1 & 0.003 & GREEN $>$ RED $=$ BLUE & chromatophore differentiation \\
\hline
\end{tabular}

For each gene, the number of transcripts compared, q-value, expression pattern and color-associated function are presented. The expression pattern represents the observed differences in mean transcript counts aggregated by gene and color morphs (see Fig. 2)

yellow, orange or red pigmentation [10,36]. Our results support an important role of pteridines in the pigmentation of red and green color morphs of O. pumilio. Importantly, the full set of eight pteridine synthesis genes were significantly more highly expressed in red versus blue and green versus blue frogs, the expected pattern if they are functionally involved in coloration differences. Of these eight, three were differentially expressed at the individual level (spr, pts, and $x d h)$ and these have all been previously implicated in variation in red to yellow skin pigmentation in studies of fishes and reptiles [12, $13,37,38]$. In particular, increased expression of spr has been related to the red morphs of the European wall lizard [37], and red frogs had the highest expression of this gene. Earlier studies on anurans identified $x d h$ as a candidate for pigmentation variation in the tree frog Agalychnis dacnicolor, where $x d h$ inhibition resulted in reduced pigmentation supporting the role of this enzyme in the synthesis of pterorhodin, a red pigment [39].
More recently, a gene expression study on captive bred Dendrobates auratus, a dendrobatid frog, reported that blue-black morph individuals show lower expression of $x d h$ transcripts than the brown or greenish-blue dorsum morphs [33]. This matches perfectly with the expression profile observed in our study and supports the role of $x d h$ in the synthesis of pteridines responsible for the red and green skin coloration in O. pumilio. Pts and spr regulate the second and third steps in the pteridine synthesis pathway while $x d h$ regulates the final production of several yellow pteridine pigments [12].

Only a few genes have previously been directly implicated in carotenoid coloration and so it is notable that two of them were differentially expressed among $O$. pumilio populations. Bco2 (Beta, beta-carotene 9,10-oxygenase) encodes a carotenoid cleaving enzyme which explains white to yellow variation in the coloration of chicken legs [40], and has also low expression in yellow wall lizards [11]. Here we found that $b c 02$ had higher 


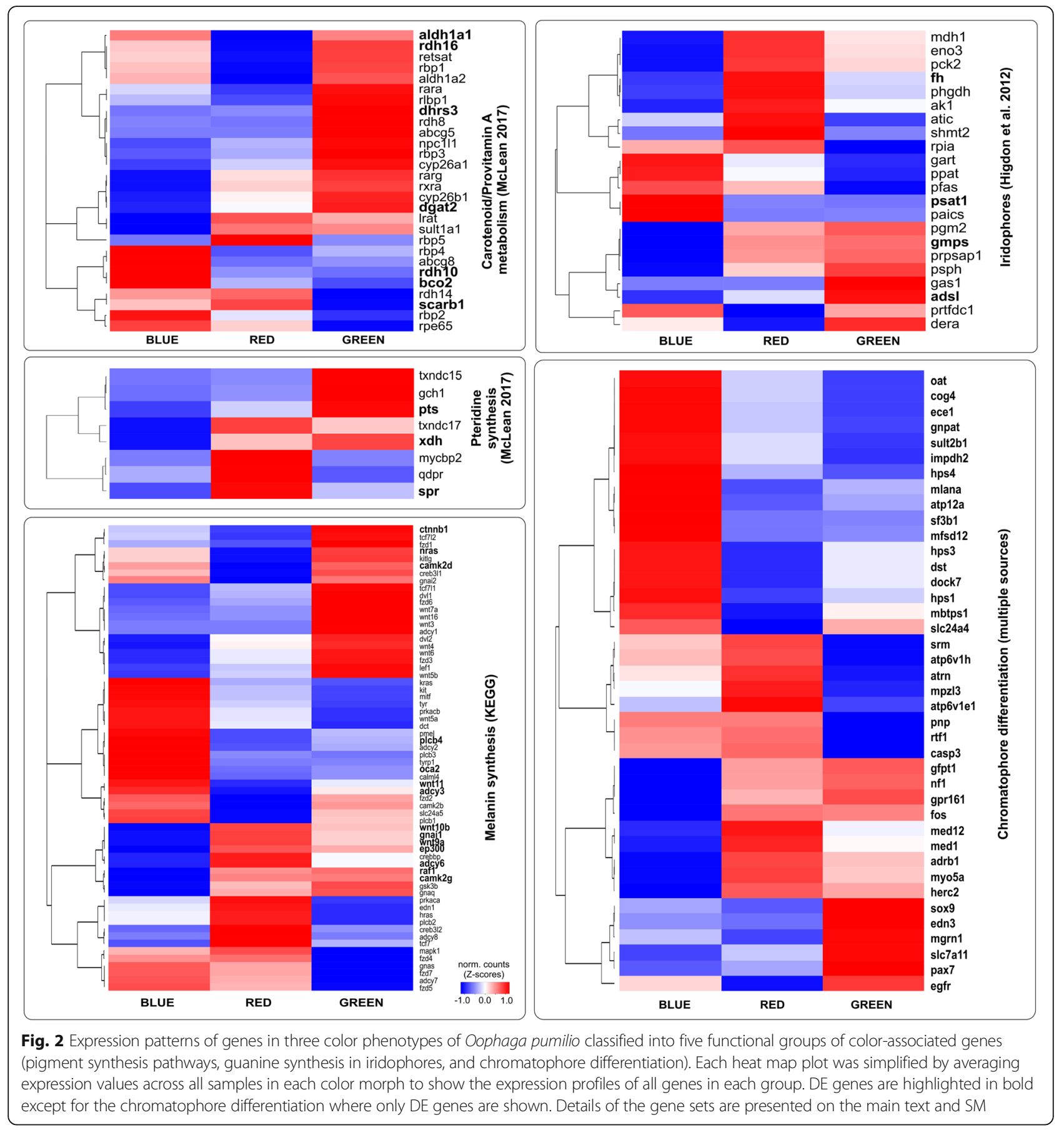

Table 2 Results of the gene-set enrichment analysis (GSEA) testing for differences in expression of color-associated gene sets between color phenotypes

\begin{tabular}{|c|c|c|c|c|}
\hline contrast & Pteridine synthesis & Carotenoid metabolism & Melanin synthesis & Guanine synthesis (Iridophore) \\
\hline RED vs BLUE & 0.026 & 0.521 & 0.618 & 0.698 \\
\hline GREEN vs BLUE & 0.032 & 0.378 & 0.507 & 0.752 \\
\hline GREEN vs RED & 0.844 & 1.000 & 0.510 & 0.635 \\
\hline
\end{tabular}

For each pair-wise comparison the false detection ratio $(F D R)$ is shown with significant results (FDR $<0.25)$ highlighted in bold. Genes included in each set and their expression profiles are illustrated in Fig. 2 


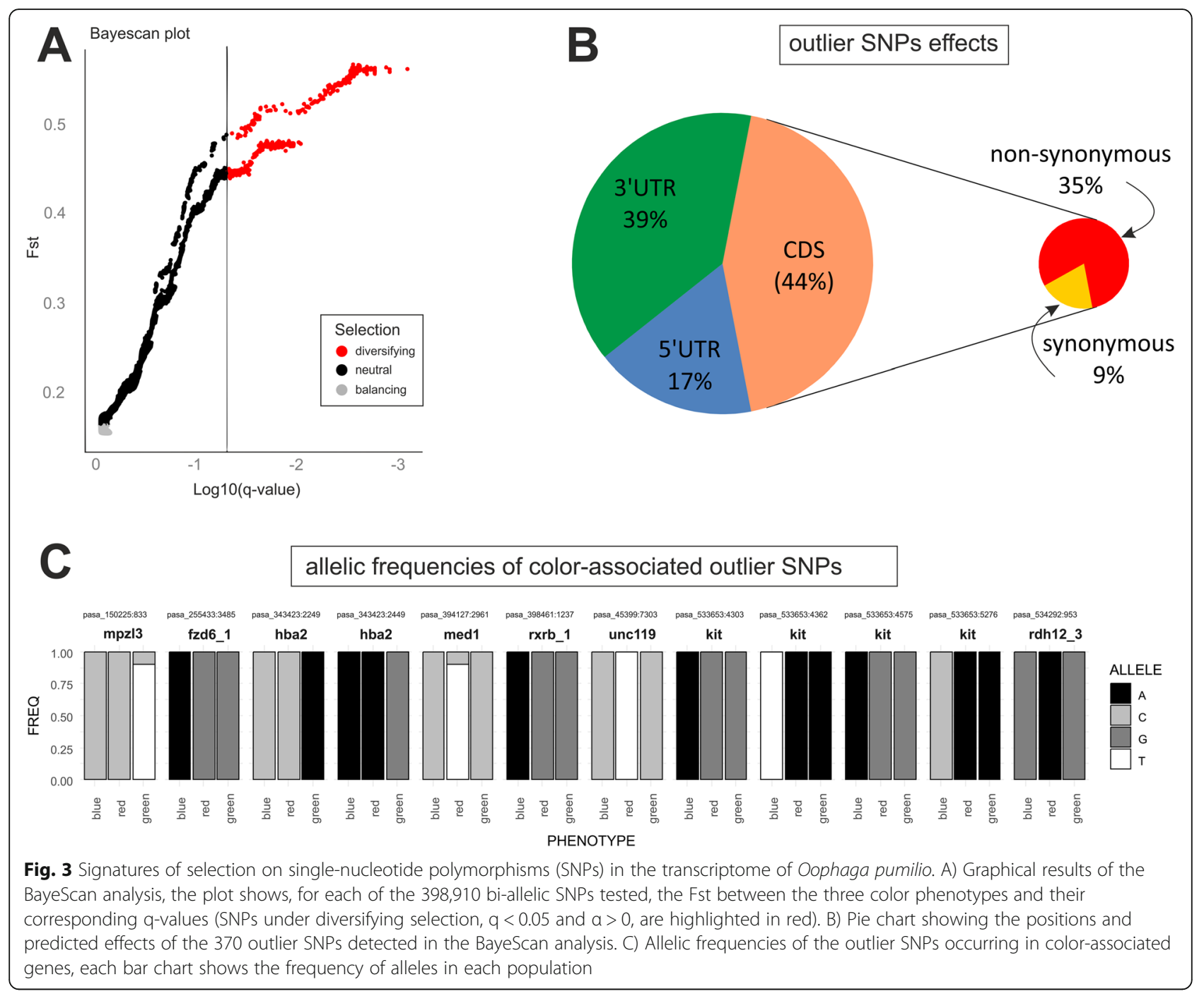

Table 3 Color-associated genes with SNPs showing signatures of directional selection among the three color phenotypes of 0. pumilio studied

\begin{tabular}{|c|c|c|c|c|c|c|}
\hline SNP & alleles & q-value & alpha & $F_{\text {st }}$ & gene & Link to pigmentation \\
\hline pasa_150225:833 & $\mathrm{C} / \mathrm{T}$ & 0.042 & 1.396 & 0.447 & mpzl3_2 & severe skin and hair abnormalities in mice $(p)$ \\
\hline pasa_255433:3485 & $\mathrm{G} / \mathrm{A}$ & 0.032 & 1.623 & 0.498 & fzd6_1 & melanin synthesis ( $p$ ) \\
\hline pasa_343423:2249 & $\mathrm{C} / \mathrm{A}$ & 0.002 & 1.916 & 0.561 & hba2 & hyperpigmented human skin \\
\hline pasa_343423:2449 & $A / G$ & 0.002 & 1.940 & 0.567 & hba2 & hyperpigmented human skin \\
\hline pasa_394127:2961 & $C / T$ & 0.046 & 1.387 & 0.445 & med1 & retinal pigmentation \\
\hline pasa_398461:1237 & $\mathrm{G} / \mathrm{A}$ & 0.006 & 1.795 & 0.535 & rxrb_1 & carotenoid metabolism (p) \\
\hline pasa_45399:7303 & $C / T$ & 0.018 & 1.718 & 0.518 & unc119 & retinal pigmentation \\
\hline pasa_533653:4303 & $\mathrm{G} / \mathrm{A}$ & 0.002 & 1.935 & 0.566 & kit & melanin synthesis \\
\hline pasa_533653:4362 & $A / T$ & 0.003 & 1.922 & 0.563 & kit & melanin synthesis \\
\hline pasa_533653:4575 & $\mathrm{G} / \mathrm{A}$ & 0.002 & 1.919 & 0.562 & kit & melanin synthesis \\
\hline pasa_533653:5276 & $\mathrm{A} / \mathrm{C}$ & 0.002 & 1.935 & 0.565 & kit & melanin synthesis \\
\hline pasa_534292:953 & $\mathrm{G} / \mathrm{A}$ & 0.007 & 1.755 & 0.526 & rdh12_3 & carotenoid metabolism (p) \\
\hline
\end{tabular}

For each SNP entry, the position and alternative alleles, as well as BayeScan results (posterior probability, q-value, alpha, $F_{\text {st }}$ ) are presented along with the gene symbol and potential link to pigmentation. Gene symbols followed by underscore and a number represent potential paralogs and their functions ( $p$ ) are less reliable 
expression in blue than green or red frogs, consistent with a potential role in cleaving carotenoids in the skin of blue frogs. The second locus, scarb1, is a carotenoid transporter involved in carotenoid uptake into cells, responsible for the white canary phenotype when its function is disrupted [41]. Again, consistent with this function, scarb1 had highest expression in red frogs. Interestingly, other genes involved in carotenoid metabolism are more highly expressed in green and blue frogs than red frogs. However those genes that are significantly higher expressed in green or blue frogs (dgat2, rhd10, rdh16, dhrs3 and aldh1a1) mostly code for enzymes of retinol metabolism which act downstream of carotenoid cleavage and are unlikely to affect carotenoid coloration [42]. We found no compelling evidence that a skin-expressed cytochrome P450 gene (CYP) is involved in production of ketocarotenoids in red frogs as occurs in red birds and turtles [43-45]. We found 30 CYP genes in the list of DE genes but except for the cyp $8 b 1$ gene (involved in bile acid synthesis and not a good candidate), all of them were potential paralogs which complicates functional interpretations. A strikingly complex mixture of carotenoids has been recently identified in skins of the orange color morph of O. pumilio [46] and further studies will be required to elucidate the particularities of carotenoid metabolism in these frogs.

The structural blue coloration in frogs likely depends on both the iridophore layer and the underlying melanophore layer and so we expected that genes affecting blue coloration should be expressed in these cells. A strong candidate from iridophores is psat1, which has higher expression in blue than red or green frogs and is in the biosynthetic pathway for guanine. When comparing our findings in Fig. 2 (heatmap) to the guanine synthesis cycle for zebrafish [47] an interesting pattern emerges: those genes which have higher expression in blue frogs are those coding for enzymes that contribute to the production of SAICAR (phosphoribosylaminoimidazolesuccinocarboxamide) from 5-Phosphoribosyl 1Pyrophosphate (PRPP) (ppat, pfas, gart, paics; purine synthesis) and prtfdc1 that directly produces guanine from guanosine monophosphate (GMP, i.e. guaninespecific), while the genes that are more highly expressed in red frogs are mainly located in the citrate cycle $(f h$, $m d h 1 a, p c k 2$, eno3) and serine/glycine pathway (phgdh, shmt2) which does not directly lead to the production of purine or guanine (except atic). There is no clear pattern for the location of the genes in the guanine synthesis cycle, which are more highly expressed in the green frogs. In summary, the blue frogs have genes upregulated that produce SAICAR, which is the starting product for purine and guanine synthesis, while red frogs have genes upregulated that recycle SAICAR for precursors of the citrate cycle, glycolysis and production of amino acids (serine, glycine). Psat1 (highly expressed in blue frogs) reconnects the serine/glycine pathway back to purine synthesis.

For melanophores, two interesting candidates that might affect blue coloration in O. pumilio are oca 2 and myo5a. These genes show an opposed expression pattern in blue frogs, the oca 2 gene shows up-regulation while the myo5a gene is downregulated. Oca2 plays an important role in melanogenesis, with mutations leading to paler coloration in fish and humans [48]. The myo5a gene encodes a protein involved in short-range movement of melanosomes along actin filaments and plays a role in moving melanosomes to the dendrites. Defects in myo5a lead to the lavender (pale grey) phenotype in many mammalian and avian species [e.g. in horses, 56], in which the transfer of melanosomes to keratinocytes occurs. In species such as frogs where this process does not occur, the phenotypic effects may be different.

Several of the differentially expressed genes involved in chromatophore development are also interesting candidates. Disruption of $s f 3 b 1$, which is upregulated in blue frogs, is responsible for proliferation of dermal melanocytes and blue coloration via the Tyndall effect in humans[49].

Blue frogs also show increased expression of the hps1, hps3, hps4 genes. These genes encode three HermanskyPudlak syndrome proteins, which in humans are linked to oculocutaneous albinism with abnormally light coloring of the skin, hair, and eyes. One of them (hps3) was also found up-regulated in all three color phenotypes of Dendrobates auratus that included blue skin patches [33]. The pax7 and sox 9 genes are two interesting candidates that showed increased expression in green frogs and might be responsible for this particular coloration. The pax7 gene plays a key role in the differentiation of xanthophore precursor cells and its absence results in a complete depletion of differentiated xanthophores in embryos as well as in adult zebrafish [50]. The expression of the $\operatorname{sox} 9$ gene is known to stimulate melanin production and leads to increased human skin pigmentation in response to UV stimulus [51].

Comparison of our gene expression results with those of Stuckert et al. [33] revealed 240 genes showing differential expression between color morphs in both species (i.e. O. pumilio and D. auratus, SMTable 6). The list includes genes related to pteridine synthesis $(x d h)$, melanogenesis (gnai1, raf1), 12 genes involved in melanophore genesis and differentiation (e.g. atp12a, hps3, and sox9). Although the color differences among the morphs differ in the two species, some of these genes may play general roles in amphibian coloration and should be the target of future studies. 


\section{SNP variation}

The results obtained at the SNP level suggest that sequence variation in multiple loci may be contributing to adaptive color variation among these frogs. The kit gene encodes a growth factor receptor protein which is known to play a key role regulating the melanin synthesis pathway as well as melanophore survival and positioning of kit receptor-expressing melanocytes [52, 53]. The existence of four variants of kit exclusive to blue frogs strongly suggest that this gene might have an important role in color determination in these frogs, a subject that should be scrutinized further with additional population surveys and laboratory studies. Interestingly, two other studies on species of the Dendrobatidae family have pointed to variants in another gene, the $m c 1 r$ gene (encoding the melanocyte-stimulating hormone receptor) as a main candidate for explaining pigmentation differences $[33,54]$. However, we did not find any variation in the mc1r gene among the color morphs studied, including the variant position in the $O$. histrionica species complex (SMFigure 5). In the two previous studies the phenotypic differences between the color morphs tested included color patterning variation, while in our study the differences are solely related to background coloration itself. This suggests that these variants in the $m c 1 r$ gene might be involved in color patterning and not related to background coloration itself, which would match the profile of the candidate color-patterning gene inferred by the cross-breeding experiments of Summers et al. [32].

The abundance of outlier SNPs landing on 3' untranslated regions (UTR) is noteworthy. Although such a pattern might arise by chance if the 3'UTRs in the dataset are longer than the 5'UTRs, inspection of feature length distribution in our reference transcriptome does not indicate that length differences could explain the abundance of SNPs in the 3'UTR regions (SMFigure 6). Polymorphisms in the 3'UTR can alter the secondary structure and affect mRNA stability [55] and these regions are also important targets of post-transcriptional regulation via miRNAs $[56,57]$.

The finding that a high proportion of outlier SNPs occurred in genes with differential expression (40\%) gives high confidence that the BayeScan analysis has uncovered functionally relevant outliers. The selection test implemented in BayeScan accounts for uncertainty in allele frequencies providing unbiased estimates even with very small sample sizes, albeit with the risk of a low power [58]. Given the small number of individuals per population, the statistical power of our implementation of BayeScan is modest and it is likely that the set of outlier SNPs identified in this study represents only a fraction of the total SNPs experiencing positive selection. On the other hand, genomic selection scans are sensitive to the strength of selection and local recombination rate as well as the underlying genetic structure, demography and migration rate of the sampled populations [59]. Characterizing and accounting for those sources of variation should be the goal of future in-depth studies on the targets of selection in these frogs.

\section{Future directions}

Our results on SNP variation and gene expression concern only those genes that are consistently expressed in the skin and hence represent a subset of all the genomic loci involved in skin pigmentation of these frogs. Additionally, SNP calling from RNA-Seq data cannot capture variation involved in untranscribed regulatory regions that may underlie the majority of adaptive phenotypic variation, unless linkage disequilibrium is high between regulatory and coding regions [60]. We are, however, confident that our results provide an extensive list of color-associated genes that represent good candidates for future insights into the genomics of color polymorphism in these frogs.

The limited genomic resources available for O. pumilio surely limit our interpretations and an adequate assembly and annotation of the genome will allow a more detailed interpretation of our results. Besides an adequate reference genome, subsequent studies should address the following goals: 1) the comparison of gene expression and SNPs among additional populations with blue, green and red dorsal coloration to find out whether similar coloration in geographically distinct localities rely on the same underlying molecular processes; 2) analyses of differential expression in additional organs that may be involved in pigment uptake and metabolism (e.g. liver and gut); 3) the inclusion of additional frog color morphs (yellow, orange, black-white) not considered in this research, and 4) the assessment of gene expression of candidate genes in hybrid populations; for example in a contact zone where red, blue and all shades of intermediate phenotypes (purple) co-occur (e.g. Yang et al. [61]).

\section{Conclusions}

The three populations of frogs exhibiting red, blue and green coloration showed highly divergent gene expression in their skins. The strong signal of differential expression in pteridine genes is consistent with a major role of these genes in generating the coloration differences among the three morphs, and is consistent with the limited data available for other amphibians. We found a total of 68 differentially expressed genes linked to pigment production, structural coloration in iridophores, and pigment-cell differentiation which suggests that multiple mechanisms are responsible for the coloration differences, likely involving both pigment-based 
and structural coloration. In addition to regulatory differences, we found evidence of differential selection acting at the DNA sequence level in several colorassociated loci, which underlines the polygenic nature of this color polymorphism. Our research provides an important landmark for future studies on the evolutionary diversification of coloration in O. pumilio and other amphibians.

\section{Methods}

\section{Sampling and sequencing}

Field work was conducted in Panama on the Bocas del Toro archipelago from March 25 to March 31 in 2015. We sampled three wild populations of $O$. pumilio: a red


W $082^{\circ} 22.051$ ), a green population on Isla Popa $\left(\mathrm{N} 09^{\circ} 08.500, \mathrm{~W} 082^{\circ} 07.615\right)$ and a blue population on the Tierra Oscura peninsula (N 09 12.685 , W $082^{\circ} 12.191$ ). We found large frog populations and captured (between 9:00 and 11:00) five adult males from each locality (15 individuals in total). The frogs were anaesthetized in Tricain S (MS222) and sacrificed by decapitation always at the same time of the day (around 13:00). The dorsal skin was immediately resected and stored in RNAlater. Capture and euthanasia procedures followed ethical guidelines for amphibian research [62] and procedures and export permits were extended by the Panamanian government (ANAM: SC/A-5-15 and SEX/A-25-15) and import permits, of fixed skins, by the Bundesamt für Naturschutz (BafN E-01676/15).

We used the RNeasy Mini Kit (Qiagen) to extract total RNA from the skin samples according to the manufacturers' instructions, with a final elution in $30 \mu \mathrm{l}$. RNA quantity and integrity was measured using a Bioanalyser (Agilent). RNA concentration varied between 106 and $363 \mathrm{ng} / \mu \mathrm{l}$ and the RNA integrity number (RIN) ranged from 8.5 to 9.4. Two $\mu$ g RNA of each sample were used to create molecularly indexed paired-end cDNA libraries, which were sequenced in $100 \mathrm{bp}$ fragments on an Illumina $\mathrm{HiSeq}^{\mathrm{Tm}} 2000$ platform. Library preparation and sequencing were performed by BGI (Shenzhen, China).

\section{Draft genome re-scaffolding}

Despite substantial advances in genome assembly algorithms, accurate genome assemblies remain challenging for organisms with large genomes where repetitive regions abound. This challenge is pervasive among amphibians and prominent in the recently assembled $O$. pumilio genome draft, which is a heavily fragmented assembly in spite of extensive and high quality sequence data [63]. In order to re-scaffold the low-quality draft genome of $O$. pumilio we used P_RNA_Scaffolder software [64]. This software, like other recently developed scaffolding algorithms, uses the information from RNA-
Seq reads to complete the structures of transcribed regions, since RNA-sequencing captures both mRNAs and long non-coding RNAs [64]. We used as input the paired-end RNA-Seq reads aligned to the original draft genome assembly. RNA-Seq reads obtained in this study derive from skin (15 individuals), liver (2 inds.), and retina ( 1 ind.). To represent additional tissues, we added the available sequences from brain and ova available at NCBI (SRA accessions: SRR8275033, SRR7639587-90, SRR7639592). Raw Illumina reads were quality-trimmed and adaptor sequences removed using Trimmomatic with default settings. Reads were mapped to the draft genome using HISAT2 [65] and P_RNA_Scaffolder was run on the resulting .bam file using default settings.

\section{De-novo reference transcriptome assembly}

The generation of a reference transcriptome is a crucial step for expression quantification and was challenging in our study as samples from different localities are likely to exhibit substantial variation in expression profiles and probably comprise different genotypes which can result in assembly artifacts [66]. We therefore generated a reference $O$. pumilio transcriptome using a combination of genome-guided and de-novo assembly strategies. For the genome-guided approach, we mapped the qualitytrimmed reads to the re-scaffolded draft genome of $O$. pumilio [63] using STAR [67] two-pass algorithm with default settings. The mapped reads were then used to generate a genome-guided transcriptome assembly in Trinity $[68,69]$ with default settings except for maximum intron size $=30 \mathrm{k}$ and minimum $\mathrm{k}$-mer coverage $=$ 2. A de novo transcriptome assembly was also generated using the quality-trimmed reads as input and identical settings in Trinity. Finally, the resulting genome-guided and de-novo transcriptome assemblies were collated into a set of non-redundant transcripts by aligning them to the O. pumilio re-scaffolded genome draft using PASA [70]. The PASA pipeline functions applied included: Transcript cleaning (identify and strip polyadenylation, trim vectors, and discard low quality transcripts); mapping and aligning transcripts to the genome (using GMAP [71]); validate nearly perfect alignments (95\% identity, over $90 \%$ of transcript length, and consensus splice sites); maximal assembly of spliced alignments clustering and assembling of valid transcript alignments based on genome mapping location. To account for the extensive fragmentation of the re-scaffolded genome draft we used the "PASA_comprehensive_db" pipeline to add to the PASA assemblies those de-novo or genomeguided assembled transcripts that align partially to the genome but extend onto sequencing gaps ( $>30 \%$ of transcript length and $95 \%$ identity).

The resulting reference transcripts were translated into amino acid sequences using Transdecoder with default 
settings and retaining transcripts with positive hits against Pfam 32.0 [72] and UniProt [73] databases. Blastp searchers were performed using Diamond [74] with $1 \times 10^{-3} \mathrm{E}$-value threshold for positive alignments. The blastp hits of the resulting set of coding transcripts were sorted by alignment score, length and E-value and the top-hit blast results were used to annotate the transcripts using Annie [75]. In order to improve functional annotation of transcripts, we searched for orthologs with Uniprot entries using reciprocal best blastp hits (RBBH). We then annotated the resulting list of ortholog transcripts with their corresponding Uniprot RBBH into a transcript-to-gene table. Other transcripts with positive blastp hits against a Uniprot gene already represented in the transcript-to-gene table but residing on a different genomic cluster (i.e, with a different PASA_cluster id) were added to this table but annotated as potential paralogs by adding numeric suffixes to their corresponding gene symbol.

\section{Differential expression analyses}

Transcript abundance in the skin samples was estimated by pseudo-aligning the quality-trimmed reads to the transcriptome reference sequences with Kallisto software [76] using 100 bootstraps for uncertainty estimation. Differential expression analyses were later conducted with the Sleuth R-package [77] by comparing the transcript abundance estimates among three groups (blue, red and green morphs) of five individuals each. We imported the transcript abundance estimates of all samples and filtered out all low expressed transcripts, with fewer than five reads in more than $30 \%$ of the samples, using a custom filter function in Sleuth. We identified differentially expressed (DE) transcripts by comparing a model including color as an explanatory variable against a null model using a likelihood ratio test (LRT). We applied the gene aggregation algorithm in Sleuth in which the $p$ values of the LRT at the transcript level are weighted by the mean expression level of the transcript and aggregated into genes following a meta-analysis approach. This method outperforms classic "aggregate first and test later" methods where the assignment of a single numerical count value to a gene can mask dynamic effects among its multiple constituent transcripts [78]. We used our transcript-to-gene table, see above, for genelevel aggregation of statistical results and considered as differentially expressed those genes with a false detection ratio $(\mathrm{FDR})<0.05$, as calculated from the LRT $p$-values after adjustment for multiple testing with the BenjaminiHochberg procedure. Sleuth calculations were run in $\mathrm{R}$ [79]. Batch effects in expression quantification were assumed negligible in our study as all samples were processed as one batch during library preparation and sequencing. An additional inspection of a PCA plot of transcript expression labeled by samples and sequencing lanes (SMFigure 7), revealed no noticeable effect of the sequencing lane.

\section{Definition of color-associated gene sets}

A large number of $\mathrm{DE}$ genes are to be expected when comparing expression profiles among samples from the natural populations in this system as genes responsive to any other factor that aligns with coloration will be flagged as DE. The resulting gene lists are then likely to include many genes of which color-associated genes will be a potentially very small fraction. In order to focus on coloration-associated genes, we constructed a list of genes potentially linked to pigment synthesis pathways or structural coloration in previous studies on various vertebrate groups. The resulting list includes 368 genes that have been previously shown to be associated with carotenoid metabolism, pteridine synthesis, melanogenesis, iridophore guanine synthesis, and chromatophore development (SMTable 7). This list is probably not exhaustive as other genes might be associated to coloration in amphibians, and particularly in these frogs, for which the mechanisms of coloration are poorly studied.

Heatmap plots to characterize the expression profiles were drawn using ComplexHeatmaps [80]. Clusters of DE genes were then identified by hierarchical clustering of expression values and functional classification of genes in each subset was performed with the aid of PANTHER [81], at the pathway function level and matching gene symbols against Хenopus tropicalis annotations.

\section{Gene set enrichment and over-representation analyses}

In order to further investigate the expression pattern among color phenotypes, we applied Gene-Set Enrichment Analysis (GSEA, [82]) to investigate whether the four color-related gene sets (carotenoid metabolism, pteridine synthesis, melanogenesis, and iridophore guanine synthesis; see SMTable 7), showed differential expression among the three color phenotypes (three groups, five individuals each). GSEA was run for each color pair comparison using the gene-level expression estimates from Kallisto as input and with default settings except for using gene set permutations and minimum size of gene set $=5$. Additionally, to characterize the functional significance of the entire set of DE genes we performed a Gene Ontology (GO) term over-representation analysis of these genes using ConsensusPathDB-human [83]. We used the entire set of annotated ortholog genes in the $O$. pumilio transcriptome as background against the over-represented GO-based sets in the DE gene list were searched for. We summarized the resulting long list of enriched gene functional categories by clustering into representative subset 
of the terms and visualizing cluster importance using a multidimensional plot, as implemented in REVIGO [84].

\section{SNP detection and quantification of signatures of selection}

To overcome the low quality of assembly and annotation of the O. pumilio draft genome, we adopted a superTranscriptome approach and used Lace software to combine the assembled transcripts into a set of super-transcripts that contain the sequence of all exons of a gene without redundancy [85]. The gene-to-transcript map, as obtained from PASA, was used as input to Lace to guide the concatenation of transcripts. The resulting superTranscriptome was used as a reference against which all RNA-Seq skin reads of the three color phenotypes were mapped using Hisat2 [65]. Single-Nucleotide Polymorphism (SNP) variants were called using GATK software, following the best-practice workflow for RNA-Seq SNP calling. This pipeline includes: align, sort and mark duplicate read mappings; split reads into exon segments and clip sequences overhanging the intronic regions (SplitNCigarReads); call variants with phred-scaled confidence threshold $>20$; filter results based on Fisher Strand values (FS $>30.0)$ and Qual By Depth values $(\mathrm{QD}<2.0)$. The resulting VCF file was further filtered using vcftools [86] to include only the bi-allelic SNPs, with a minor allele frequency $>0.033$ (more than one in 15 diploid individuals), genotyped with $>10 \mathrm{X}$ coverage and $<50 \%$ missing data across individuals. We used the resulting genotypes to identify SNPs showing signatures of selection among the blue, red and green color phenotypes of $O$. pumilio (three groups, five individuals each) using BayeScan software [87]. The required input file was prepared using the $\mathrm{R}$ package radiator (https://github.com/thierrygosselin/radiator). BayeScan was run for 100,000 iterations $(50,000$ discarded as burn-in) and sampled every 10th iteration, with a prior odds value of 10 and a false discovery rate (FDR) of 0.05 . Twenty pilot runs were used to choose the proposal distribution for the reversible jump MCMC algorithm, convergence of the run was assessed from the values of effective sizes and visual inspection of the traces of the sampled output using the coda R-package. We mapped the PASA comprehensive collection of transcripts to super-transcriptome using GMAP [71] and predicted the functional impact of the outlier SNPs on the transcript structures using SNPdat [88].

\section{Supplementary information}

Supplementary information accompanies this paper at https://doi.org/10. 1186/s12864-020-6719-5.

Additional file 1 SMTable 1: Assembly statistics of the original Oophaga pumilio draft genome and the re-scaffolded draft produced for this study.
Additional file 2 SMTable 2: Results of the differential expression analysis between blue, green and red color phenotypes of 0 . pumilio, obtained using Sleuth software. For each gene, the number of transcripts compared, q-value, expression pattern and color-associated function are presented. Putative paralogs are denoted in the symbol column by a "_" followed by a number.

Additional file $\mathbf{3}$ SMTable 3: Results of the functional classification of gene expression clusters at the pathway level. The results were obtained in PANTHER using the symbols of the differentially expressed genes mapped against the Xenopus tropicalis annotation.

Additional file 4 SMTable 4: Results of the over-representation analysis of the 1445 genes found to be differentially expressed between color phenotypes of $O$. pumilio frogs. Analysis was conducted using ConsensusPathDB. GO category names whose hypergeometric $p$-value $<0.01$ are listed, and for each set, the set size (the absolute size, as well as the corrected size), the over-representation $\mathrm{p}$-value, the q-value (false discovery rate), the set size and all input genes in each set are provided.

Additional file $\mathbf{5}$ SM Table 5: Annotated SNPs showing signatures of positive selection in BayeScan analyses. Putative paralogs are denoted in the symbol column by a "_" followed by a number. Functional impact of SNPS was evaluated on the transcripts of each gene using SNPdat. SNPs on genes that also showed significant expression differences are highlighted in bold (see main text for further details).

Additional file 6 SM Table 6: Differentially expressed genes in skin samples of different color phenotypes of Oophaga pumilio and Dendrobates auratus [33].

Additional file 7 SM Table 7: List of the color-associated genes, compiled from the literature and used for functional interpretation.

Additional file 8 Supplementary figures.

\section{Abbreviations}

adcy6: Adenylate cyclase type 6; adsl: Adenylosuccinate lyase;

aldh1a1: Retinal dehydrogenase 1; atic: Bifunctional purine biosynthesis protein PURH; atp 12a: Potassium-transporting ATPase alpha chain 2;

bco2: Beta,beta-carotene 9',10'-oxygenase; BUSCO: Benchmarking universal single-copy ortholog genes; camk2g: Calcium/calmodulin-dependent protein kinase type II subunit gamma; CDS: Coding sequences; ctnnb1: Catenin beta1; CYP: Cytochrome P 450 family genes; cyp861: 5-beta-cholestane-3-alpha,7alpha-diol 12-alpha-hydroxylase; DE: Diferencially expressed;

dgat2: Diacylglycerol O-acyltransferase 2; dhrs3: Short-chain dehydrogenase/ reductase 3; dock7: Dedicator of cytokinesis protein 7; eno3: Beta-enolase; ep300: Histone acetyltransferase p300; fh: Fumarate hydratase, mitochondrial; gart: Trifunctional purine biosynthetic protein adenosine-3; gnai1: Guanine nucleotide-binding protein G(i) subunit alpha-1; GO: Gene Ontology term; GTP: Guanosine triphosphate; hps 1: Hermansky-Pudlak syndrome 1 protein homolog; hps3: Hermansky-Pudlak syndrome 3 protein; hps4: HermanskyPudlak syndrome 4 protein; mdh $1 a$ : Malate dehydrogenase, cytoplasmic; med1: Mediator of RNA polymerase II transcription subunit 1;

med12: Mediator of RNA polymerase II transcription subunit 12; myo5a: Unconventional myosin-Va; oca2: P protein; paics: Multifunctional protein ADE2; pax7: Paired box protein Pax-7; pck2: Phosphoenolpyruvate carboxykinase [GTP], mitochondrial; pfas: Phosphoribosylformylglycinamidine synthase; phgdh: D-3-phosphoglycerate dehydrogenase; plcb4: 1phosphatidylinositol 4,5-bisphosphate phosphodiesterase beta-4; ppat: Amidophosphoribosyltransferase; PRPP: 5-Phosphoribosyl 1Pyrophosphate; prtfdc1: Phosphoribosyltransferase domain-containing protein 1; psat1: Phosphoserine aminotransferase; pts: 6-pyruvoyl tetrahydrobiopterin synthase; raf1: RAF proto-oncogene serine/threonine-protein kinase; rdh10: Retinol dehydrogenase 10; rdh16: Retinol dehydrogenase 16; RNA: Ribonucleic acid; SAICAR: phosphoribosylaminoimidazolesuccinocarboxamide; scarb1: Scavenger receptor class B member 1: sf3b1: Splicing factor 3B subunit 1; shmt2: Serine hydroxymethyltransferase, mitochondrial; SNP: Single nucleotide polymorphism; sox7: Transcription factor Sox-7; spr: Sepiapterin reductase; UTR: Untranslated regions; wnt 10b: Protein Wnt-10b; wnt9a: Protein Wnt-9a; $x d h$ : Xanthine dehydrogenase/oxidase 


\section{Acknowledgements}

We thank Ricardo Cossio for help during field work and acknowledge the support received from the staff of the Smithsonian Bocas del Toro Research Station in Panamá. We are also grateful to Sönke von den Berg for help with map layout. Computations were performed at the zentrale Informations- und Datenverarbeitungsservice der Stiftung TiHo Hannover (TiHo-IDS) and kindly facilitated by Silvester Jordan and Frank Burkhart.

\section{Authors' contributions}

$\mathrm{HP}, \mathrm{RI}$ and $\mathrm{NM}$ designed the study. $\mathrm{HP}, \mathrm{RI}$ and $\mathrm{NM}$ conducted the field work. AR conducted bioinformatic analyses. AR, HP and NM drafted the manuscript, and all authors read, corrected and approved the final manuscript.

\section{Funding}

This study was funded by a grant from the Deutsche Forschung Gemeinschaft (DFG: PR 626/6-2 to HP). RI work was supported by the Sistema Nacional de Investigación, the Panama Amphibian Rescue and Conservation Project, and Minera Panamá. The funders had no role on study design, data acquisition and analysis, or manuscript preparation.

\section{Availability of data and materials}

The raw sequencing reads have been deposited in NCBI Sequence Read Archive (under bioproject: PRJNA610154). Additional reads from previous studies, used to assemble the reference transcriptome, are available at NCBI (SRA accessions: SRR8275033, SRR7639587-90, SRR7639592). The newly assembled comprehensive transcriptome of Oophaga pumilio is available in the Transcriptome Shotgun Assembly (TSA) database (accession: GIKS00000000). The re-scaffolded genome and the super transcriptome of Oophaga pumilio obtained in this study are available at Zenodo [89]. The datasets supporting the conclusions of this article are included within the article and eight additional files.

\section{Ethics approval and consent to participate}

Fieldwork and animal use for this research was approved by the National Environmental Authority of Panamá (ANAM: SC/A-5-15). Capture and euthanasia procedures followed ethical guidelines for amphibian research [62] and procedures and export permits were extended by the Panamanian government (ANAM: SC/A-5-15 and SEX/A-25-15) and import permits, of fixed skins, by the Bundesamt für Naturschutz (BafN E-01676/15).

\section{Consent for publication}

Not applicable.

\section{Competing interests}

None.

\section{Author details}

${ }^{1}$ Institute of Zoology, University of Veterinary Medicine of Hannover, Bünteweg 17, 30559 Hannover, Germany. ${ }^{2}$ Department of Zoology, University of Cambridge, Downing St, Cambridge CB2 3EJ, England. ${ }^{3}$ Smithsonian Tropical Research Institute, Apartado Postal, 0843-03092 Panamá, República de Panamá. "Sistema Nacional de Investigación, Secretaría Nacional de Ciencia, Tecnología e Innovación, Apartado, 0816-02852 Panamá, República de Panamá.

\section{Received: 6 December 2019 Accepted: 5 April 2020 Published online: 15 April 2020}

\section{References}

1. Dreiss AN, S. A, Burri R, Fumagalli L, Sonnay C, Frey C, Goudet J, Roulin A. Local adaptation and matching habitat choice in female barn owls with respect to melanic coloration. J Evol Biol. 2012;25:103-14.

2. Brusa O, Bellati A, Meuche I, Mundy NI, Pröhl H. Divergent evolution in the polymorphic granular poison-dart frog, Oophaga granulifera: genetics, coloration, advertisement calls and morphology. J Biogeogr. 2013;40:394408.

3. Jablonski NG, Chaplin G. The colours of humanity: the evolution of pigmentation in the human lineage. Phil Trans R Soc B. 2017;372:20160349.

4. Endler JA, Mappes J. The current and future state of animal coloration research. Phil Trans R Soc B. 2017;372:20160352.
5. Hubbard JK, Safran RJ, Uy JAC, Hauber ME, Hoekstra HE. Vertebrate pigmentation: from underlying genes to adaptive function. Trends Ecol Evol. 2010;26(5):231-9.

6. Bradbury JW, Vehrencamp SL. Light and visual signal production. In: Principles of animal communication. Sunderland, MA: Sinauer Associates, Inc; 2001:113-52.

7. Caro T, Stoddard MC, Stuart-Fox D. Animal coloration research: why it matters. Phil Trans R Soc B. 2017:372:20160333.

8. Shawkey MD, D'Alba L. Interactions between colour-producing mechanisms and their effects on the integumentary colour palette. Phil Trans R Soc B. 2017:372:20160536.

9. San-Jose LM, Roulin A. Genomics of coloration in natural animal populations. Phil Trans R Soc B. 2017;372:20160337.

10. Bagnara JT. Cytology and cytophysiology of non-melanophore pigment cells. Int Rev Cytol. 1966;20:173-205.

11. Bagnara JT, Taylor JD, Hadley ME. The dermal chromatophore unit. J Cell Biol. 1968;38:67-79.

12. Ziegler I. The pteridine pathway in Zebrafish: regulation and specification during the determination of neural crest cell-fate. Pigment Cell \& Melanoma Research. 2003;16:172-82.

13. Braasch I, Schartl M, Volff J-N. Evolution of pigment synthesis pathways by gene and genome duplication in fish. BMC Evol Biol. 2007;7(74): https://doi. org/10.1186/1471-2148-1187-1174.

14. Goodwin TW. The biochemistry of the carotenoids: volume II, animals. London: Chapman \& Hall; 1984.

15. Svensson PA, Wong BBM. Carotenoid-based signals in behavioural ecology: a review. Behaviour. 2011;148(3):131-89.

16. Britton G, Liaaen-Jensen S, Pfander H (eds.): Carotenoids: Birkhäuser Verlag, Basel - Boston - Berlin; 2008.

17. Wang IJ, Shaffer HB. Rapid color evolution in an aposematic species: a phylogenetic analysis of color variation in the strikingly polymorphic strawberry poison-dart frog. Evolution. 2008;62(11):2742-59.

18. Galindo-Uribe D, Sunyer J, Pröhl H, Hauswaldt JS, Vences M. Colour and pattern variation and Pleistocene phylogeographic origin of the strawberry poison frog, Oophaga pumilio, in Nicaragua. Salamandra. 2014;50(4):225-35.

19. Summers K, Cronin TW, Kennedy T. Variation in spectral reflectance among populations of Dendrobates pumilio, the strawberry poison frog, in the Bocas del Toro archipelago, Panama. J Biogeogr. 2003;30:35-53.

20. Saporito RA, Donnelly MA, Spande TF, Garraffo HM. A review of chemical ecology in poison frogs. Chemoecology. 2011;22(3):159-68.

21. Daly JW, Spande TF, Garraffo HM. Alkaloids from . amphibian skin: a tabulation of over eight-hundred compounds. J Nat Prod. 2005;68:1556-75.

22. Daly JW, Myers CW. Toxicity of Panamanian poison frogs (Dendrobates): some biological and chemical aspects. Science. 1967;156(3777):970-3.

23. Summers K, Bermingham E, Weigt L, McCafferty S, Dahistrom L. Phenotypic and genetic divergence in three species of dart-poison frogs with contrasting parental behavior. J Hered. 1997;88(1):8-13.

24. Reynolds RG, Fitzpatrick BM. Assortative mating in poison-dart frogs based on an ecologicaly important trait. Evolution. 2007;61(9):2253-9.

25. Maan ME, Cummings ME. Sexual dimorphism and directional sexual selection on aposematic signals in a poison frog. Proc Natl Acad Sci U S A. 2009;106:19072-7.

26. Maan ME, Cummings ME. Female preferences for aposematic signal components in a polymorphic poison frog. Evolution. 2008;62(9):2334-45.

27. Dreher $\mathrm{CE}$, Pröhl $\mathrm{H}$. Multiple sexual signals: calls over colors for mate attraction in an aposematic, color-diverse poison frog. Front Ecol Evol. 2014; 2(22):e22.

28. Summers K, Symula R, Clough M, Cronin T. Visual mate choice in poison frogs. Proc R Soc B. 1999;266:2141-5.

29. Maan ME, Cummings ME. Poison frog colors are honest signals of toxicity, particularly for bird predators. Am Nat. 2012;179(1):e1-14.

30. Gehara M, Summers K, Brown JL. Population expansion, isolation and selection: novel insights on the evolution of color diversity in the strawberry poison frog. Evol Ecol. 2013;27(4):797-824.

31. Yang Y, Servedio MR, Richards-Zawacki CL. Imprinting sets the stage for speciation. Nature. 2019;574:99-102.

32. Summers K, Cronin TW, Kennedy T. Cross-breeding of distinct color morphs of the strawberry poison frog (Dendrobates pumilio) from the Bocas del Toro archipelago, Panama J Herpetol. 2004;38(1):1-8.

33. Stuckert AMM, Moore E, Coyle KP, Davison I, MacManes MD, Roberts R, Summers K. Variation in pigmentation gene expression is associated with 
distinct aposematic color morphs in the poison frog, Dendrobates auratus BMC Evol Biol 2019;19:85.

34. Grether GF, Kolluru GR, Nersissian K. Individual colour patches as multicomponent signals. Biol Rev. 2004;79:583-610.

35. Kelsh RN. Genetics and evolution of pigment patterns in fish. Pigment Cell \& Melanoma Research. 2004;17:326-36.

36. Bagnara JT, Obika M. Comparative aspects of integumental pteridine distribution among amphibians. Comp Biochem Physiol. 1965;15:33-49.

37. Andrade P, Pinho C. Lanuza GP, Afonso S, Brejcha J, Rubin C-J, Wallerman O, Pereira P, Sabatino SJ, Bellati A et al. Regulatory changes in pterin and carotenoid genes underlie balanced color polymorphisms in the wall lizard. Proc Natl Acad Sci U S A. 2019;116(12):5633-42.

38. McLean CA, Lutz A, Rankin KJ, Stuart-Fox D, Moussalli A. Revealing the biochemical and genetic basis of colour variation in a polymorphic lizard. Mol Biol Evol. 2017; 34(8):1924-35.

39. Frost SKV. Developmental aspects of pigmentation in the Mexican leaf frog Pachymedusa dacnicolor: PhD. University of Arizona; 1978.

40. Eriksson J, Larson G, Gunnarsson U, Bed'hom B, Tixier-Boichard M, Strömstedt L, Wright $D$, Jungerius $A$, Vereijken $A$, Randi $E$, et al. Identification of the yellow skin gene reveals a hybrid origin of the domestic chicken. PLoS Genet. 2008;4(2):e1000010.

41. Toomey MB, Lopes RJ, Araújo PM, Johnson JD, Gazda MA, Afonso S, Mota PG, Koch RE, Hill GE, Corbo JC, et al. High-density lipoprotein receptor SCARB1 is required for carotenoid coloration in birds. Proc Natl Acad Sci U S A. 2017;114(20):5219-24.

42. Waagmeester A, Pezik P, Coort S, Tourniaire F, Evelo C, Rebholz-Schuhmann D. Pathway enrichment based on text mining and its validation on carotenoid and vitamin A metabolism. OMICS A J Integ Biol. 2009;13(5).

43. Mundy NI, Stapley J, Bennison C, Tucker R, Twyman H, Kim K-W, Burke T, Birkhead TR, Andersson S, Slate J. Red carotenoid coloration in the Zebra finch is controlled by a cytochrome P450 gene cluster. Curr Biol. 2016;26: 1435-40.

44. Twyman H, Valenzuela N, Literman R, Andersson S, Mundy NI. Seeing red to being red: conserved genetic mechanism for red cone oil droplets and cooption for red coloration in birds and turtles. Proc R Soc B. 2016;283: e20161208.

45. Lopes RJ, Johnson JD, Toomey MB, Ferreira MS, Araujo PM, Melo-Ferreira J, Andersson L, Hill GE, Corbo JC, Carneiro M. Genetic basis for red coloration in birds. Curr Biol. 2016;26:1427-34.

46. Crothers L, Saporito RA, Yeager J, Lynch K, Friesen C, Richards-Zawacki CL, McGraw K, Cummings M. Warning signal properties covary with toxicity but not testosterone or aggregate carotenoids in a poison frog. Evol Ecol. 2016; 30:601-21.

47. Higdon CW, Mitra RD, Johnson SL. Gene expression analysis of Zebrafish melanocytes, iridophores, and retinal pigmented epithelium reveals indicators of biological function and developmental origin. PLoS One. 2013; 8(7):e67801.

48. Protas ME, Hersey C, Kochanek D, Zhou Y, Wilkens H, Jeffery WR, Zon LI, Borowsky R, Tabin CJ. Genetic analysis of cavefish reveals molecular convergence in the evolution of albinism. Nat Genet. 2006;38(1):107-11.

49. Griewank KG, Müller H, Jackett LA, Emberger M, Möller I. Nes JAvd, Zimmer L, Livingstone E, Wiesner T, Scholz SL et al. SF3B1 and BAP1 mutations in blue nevus-like melanoma. Mod Pathol. 2017;30:928-39.

50. Nord H, Dennhag N, Mucka J, Hofsten Jv. Pax7 is required for establishment of the xanthophore lineage in zebrafish embryos. Mol Biol Cell. 2016;27(11): 1853-62.

51. Passeron T, Valencia JC, Bertolotto C, Hoashi T, Pape EL, Takahashi K, Ballotti $\mathrm{R}$, Hearing VJ. SOX9 is a key player in ultraviolet B-induced melanocyte differentiation and pigmentation. Proc Natl Acad Sci U S A. 2007;104(35): 13984-9.

52. Kelsh RN, Harris ML, Colanesi S, Erickson CA. Stripes and belly-spots-a reviewof pigment cell morphogenesis in vertebrates. Semin Cell Dev Biol. 2009;20:90-104.

53. Wehrle-Haller B. The role of kit-ligand in melanocyte development and epidermal homeostasis. Pigment Cell Res. 2003;16:287-96.

54. Posso-Terranova A, Andrés JÁ. Diversification and convergence of aposematic phenotypes: truncated receptors and cellular arrangements mediate rapid evolution of coloration in harlequin poison frogs. Evolution. 2017;77(11):2677-92.

55. Day DA, Tuite MF. Post-transcriptional gene regulatory mechanisms in eukaryotes: an overview. J Endocrinol. 1998;157:361-71.
56. Skeeles LE, Fleming $J$, Mahler KL, Toland AE. The impact of $3^{\prime} U T R$ variants on differential expression of candidate cancer susceptibility genes. PLoS One. 2013;8(3):e58609.

57. Lai EC. Micro RNAs are complementary to 3' UTR sequence motifs that mediate negative post-transcriptional regulation. Nat Genet. 2002;30: 363-4.

58. BayeScan v2. 1 user manual [http://cmpg.unibe.ch/software/BayeScan/files/ BayeScan2.1_manual.pdf].

59. Weigand $H$, Leese $F$. Detecting signatures of positive selection in non-model species using genomic data. Zool J Linnean Soc. 2018;184: 528-83.

60. Hoban S, Kelley JL, Lotterhos KE, Antolin MF, Bradburd G, Lowry DB, Poss ML, Reed LK, Storfer A, Whitlock MC. Finding the genomic basis of local adaptation: pitfalls, practical solutions, and future directions. Am Nat. 2016; 188:379-97.

61. Yang Y, Blomenkamp S, Dugas MB, Richards-Zawacki CL, Pröhl H. Mate choice versus mate preference: inferences about color-assortative mating differ between field and lab assays of poison frog behavior. Am Nat. 2019; 193(4):598-607.

62. Alworth LC, Harvey SB. IACUC issues associated with amphibian research. ILAR J. 2007:48(3):278-89.

63. Rogers RL, Zhou L, Chu C, Márques R, Corl A, Linderoth T, Freeborn L, MacManes MD, Xiong Z, Zheng J, et al. Genomic takeover by transposable elements in the strawberry poison frog. Mol Biol Evol. 2018;35(12):2913-27.

64. Zhu B-H, Xiao J, Xue W, Xu G-C, Sun M-Y, Li J-T. P_RNA_scaffolder: a fast and accurate genome scaffolder using paired-end RNA-sequencing reads. BMC Genomics. 2018;19:e175.

65. Kim D, Langmead B, Salzberg SL. HISAT: a fast spliced aligner with low memory requirements. Nat Met. 2015;12(4):357-60.

66. Vijay N, Poelstra JW, Kunstner A, Wolf JBW. Challenges and strategies in transcriptome assembly and differential gene expression quantification. A comprehensive in silico assessment of RNA-seq experiments. Mol Ecol Res. 2013:22:620-34

67. Dobin A, Davis CA, Zaleski C, Schlesinger F, Drenkow J, Chaisson M, Batut P, Jha S, Gingeras TR. STAR: ultrafast universal RNA-seq aligner. Bioinformatics. 2012;29(1):15-21.

68. Grabherr MG, Haas BJ, Yassour M, Levin JZ, Thompson DA, Amit I, Adiconis X, Fan L, Raychowdhury R, Zeng Q, et al. Trinity: reconstructing a full-length transcriptome without a genome from RNA-Seq data. Nat Biotechnol. 2011; 29(7):644-52.

69. Haas BJ, Papanicolaou A, Yassour M, Grabherr M, Blood PD, Bowden J, Couger MB, Eccles D, Li B, Lieber M, et al. De novo transcript sequence reconstruction from RNA-Seq: reference generation and analysis with trinity. Nat Protoc. 2013:8:1494-512.

70. Haas BJ, Delcher AL, Mount SM, Wortman JR, Jr RKS, Hannick LI, Maiti R, Ronning CM, Rusch DB, Town CD et al: Improving the Arabidopsis genome annotation using maximal transcript alignment assemblies. Nucleic Acids Res 2003, 31(19):5654-5666.

71. Wu TD, Watanabe CK. GMAP: a genomic mapping and alignment program for mRNA and EST sequences. Bioinformatics. 2005;21(9):1859-75.

72. El-Gebali S, Mistry J, Bateman A, Eddy SR, Luciani A, Potter SC, Qureshi M, Richardson LJ, Salazar GA, Smart A, et al. The Pfam protein families database in 2019. Nucleic Acids Res. 2019;47(D1):D427-32.

73. The UniProt Consortium. UniProt: a worldwide hub of protein knowledge. Nucleic Acids Res. 2019;47:D506-D515.

74. Buchfink B, Xie C, Huson DH. Fast and sensitive protein alignment using DIAMOND. Nat Met. 2015;12:59-60.

75. Tate R, Hall B, DeRego T, Geib S. Annie: the ANNotation Information Extractor (1.0). [http://genomeannotation.github.io/annie]. Accessed on 2018.

76. Bray NL, Pimentel H, Melsted P, Pachter L. Near-optimal probabilistic RNAseq quantification. Nat Biotechnol. 2016;34(8):525-7.

77. Pimentel H, Bray NL, Puente S, Melsted P, Pachter L. Differential analysis of RNA-Seq incorporating quantification uncertainty. Nat Met. 2017; 14(7):687-90.

78. Yi L, Pimentel H, Bray NL, Pachter L. Gene-level differential analysis at transcript-level resolution. Genome Biol. 2018;19:53. https://doi.org/10.1186/ s13059-13018-11419-z.

79. R Development Core Team: R: A language and environment for statistical computing (3.5.1). [http://www.R-project.org]. Accessed on July 2018. 
80. Gu Z, Eils R, Schlesner M. Complex heatmaps reveal patterns and correlations in multidimensional genomic data. Bioinformatics. 2016;32(18): 2847-9.

81. Mi H, Muruganujan A, Huang X, Ebert D, Mills C, Guo X, Thomas PD. Protocol update for large-scale genome and gene function analysis with the PANTHER classification system (v.14.0). Nat Protoc. 2019;14:703-21.

82. Subramanian A, Tamayo P, Mootha VK, Mukherjee S, Ebert BL, Gillette MA, Paulovich A, Pomeroy SL, Golub TR, Lander ES, et al. Gene set enrichment analysis: a knowledge-based approach for interpreting genome-wide expression profiles. Proc Natl Acad Sci U S A. 2005;102(43):15545-50.

83. Kamburov A, Pentchev K, Galicka H, Wierling C, Lehrach H, Herwig R. ConsensusPathDB: toward a more complete picture of cell biology. Nucleic Acids Res. 2011;39:D712-7.

84. Supek F, Bosnjak M, Skunca N, Smuc T. REVIGO Summarizes and visualizes long lists of Gene Ontology terms. PLOS ONE. 2011;6(7):e21800: 21801-09.

85. Davidson NM, Hawkins ADK, Oshlack A. SuperTranscripts: a data driven reference for analysis and visualisation of transcriptomes. Genome Biol. 2017;18:e148.

86. Danecek P, Auton A, Abecasis G, Albers CA, Banks E, DePristo MA, Handsaker RE, Lunter G, Marth GT, Sherry ST, et al. The variant call format and VCFtools. Bioinformatics. 2011;27(15):2156-8.

87. Foll M, Gaggiotti O. A genome-scan method to identify selected loci appropriate for both dominant and codominant markers: a Bayesian perspective. Genetics. 2008;180:977-93.

88. Doran AG, Creevey CJ. Snpdat: easy and rapid annotation of results from de novo snp discovery projects for model and non-model organisms. BMC Bioinformatics. 2013;14:45.

89. Rodríguez A, Mundy NI, Ibáñez R, Pröhl H: Re-scaffolded genome and supertranscriptome of the strawberry poison frog (Oophaga pumilio) [Dataset] [https://zenodo.org/record/3696842]. Accessed on 2020

\section{Publisher's Note}

Springer Nature remains neutral with regard to jurisdictional claims in published maps and institutional affiliations.

Ready to submit your research? Choose BMC and benefit from:

- fast, convenient online submission

- thorough peer review by experienced researchers in your field

- rapid publication on acceptance

- support for research data, including large and complex data types

- gold Open Access which fosters wider collaboration and increased citations

- maximum visibility for your research: over $100 \mathrm{M}$ website views per year

At $\mathrm{BMC}$, research is always in progress.

Learn more biomedcentral.com/submissions 